\title{
CONSTRUCTION OF SCHAUDER DECOMPOSITION ON BANACH SPACES OF PERIODIC FUNCTIONS
}

\author{
by SAY SONG GOH, S. L. LEE, ZUOWEI SHEN and W. S. TANG
}

(Received 8th December 1995)

\begin{abstract}
This paper deals with Schauder decompositions of Banach spaces $X_{2 x}$ of $2 \pi$-periodic functions by projection operators $P_{k}$ onto the subspaces $V_{k}, k=0,1, \ldots$, which form a multiresolution of $X_{2 x}$. The results unify the study of wavelet decompositions by orthogonal projections in the Hilbert space $L_{2 \pi}^{2}$ on one hand and by interpolatory projections in the Banach space $C_{2 \pi}$ on the other. The approach, using "orthogonal splines", is constructive and leads to the construction of a Schauder decomposition of $X_{2 x}$ and a biorthogonal system for $X_{2 x}$ and its dual $X_{2 \times}^{*}$. Decomposition and reconstruction algorithms are derived from the construction.
\end{abstract}

1991 Mathematics subject classification: 41A65, 42C15, 46B15, 47A58.

\section{Introduction}

For $1 \leq p<\infty$, let $L_{2 \pi}^{p}$ be the Banach space of all complex-valued $2 \pi$-periodic measurable functions $f$ defined on $\mathbf{R}$ such that $\|f\|_{p}=\left(\frac{1}{2 \pi} \int_{0}^{2 \pi}|f(x)|^{p} d x\right)^{1 / p}<\infty, C_{2 \pi}$ be the space of all continuous complex-valued $2 \pi$-periodic functions on $\mathbf{R}$ and $X_{2 \pi}$ denote either $L_{2 \pi}^{p}$ or $C_{2 \pi}$. More generally, let $B \subseteq L_{2 \pi}^{1}$ be a homogeneous Banach space on $\mathbf{T}=[0,2 \pi]$ (see [8, pp. 14-15]), i.e., let $B$ be a linear subspace of $L_{2 \pi}^{1}$ with a norm $\|\cdot\|_{B}$ such that

B1 $\left(B,\|\cdot\|_{B}\right)$ is a Banach space and $\|\cdot\|_{B} \geq\|\cdot\|_{1}$,

B2 if $f \in B$ and $t \in \mathbf{T}$, then $f(\cdot-t) \in B$ and $\|f(\cdot-t)\|_{B}=\|f\|_{B}$,

B3 for all $f \in B$ and all $t \in T, \lim _{t \rightarrow 0}\|f(\cdot-t)-f\|_{B}=0$.

Suppose that for $k=0,1, \ldots$, there is a function $\phi_{k} \in B$ with the following properties:

MR1 $\mathcal{F}_{k}:=\left\{T_{k}^{\ell} \phi_{k}: \ell=0,1, \ldots, 2^{k}-1\right\}$ is linearly independent, where $T_{k}^{\ell} f:=f\left(\cdot-\frac{2 \pi}{2^{k}}\right)$,

MR2 $V_{k} \subset V_{k+1}, k=0,1, \ldots$, where $V_{k}:=\operatorname{span}\left(\mathcal{F}_{k}\right)$,

MR3 $\overline{U_{k \geq 0} V_{k}}=B$, where the closure is taken with respect to the norm $\|\cdot\|_{B}$ on $B$.

If MR1-MR3 are satisfied, we shall call $\left\{V_{k}\right\}_{k \geq 0}$ a multiresolution of $B$. We shall also say that $\phi_{k}, k \geq 0$, generate a multiresolution of $B$. The functions $\phi_{k}$ will be called scaling functions. 
Multiresolutions and wavelets in Hilbert spaces, for instance, $L^{2}(\mathbf{R})$ and $L_{2 \pi}^{2}$, are associated with orthogonal projections onto the multiresolution subspaces $V_{k}$ (see $[3,6$, $9,10,13,14])$. For each $k$, the orthogonal projection $P_{k} f$ of a function $f$ in the Hilbert space is the least squares approximant of $f$. The orthogonal projection operator $P_{k}$ also gives rise to the orthogonal decomposition $V_{k+1}=V_{k} \oplus^{\perp} W_{k}$ where $V_{k} \perp W_{k}$. One of the main problems in the study of wavelets is the construction of orthonormal bases for the orthogonal subspaces $W_{k}$. The entire collection of the orthonormal wavelet bases for $W_{k}, k=0,1, \ldots$, then forms a wavelet basis for the Hilbert space.

Whereas the least squares approximation is a convenient and efficient way of approximating functions, interpolation is very often more desirable in practice. Interpolation operators are projection operators. For a multiresolution of the space $C_{u}(\mathbf{R})$ of bounded uniformly continuous functions on $\mathbf{R}$, an interpolation operator $I_{k}: C_{u}(\mathbf{R}) \rightarrow V_{k}$ decomposes $V_{k+1}$ into an algebraic direct sum $V_{k+1}=V_{k} \oplus W_{k}$ where $V_{k} \cap W_{k}=\{0\}$. In this case the interpolating wavelet bases for the algebraic complements $W_{k}$ have been studied by Micchelli [12], Donoho [7] and Chui and Li [2].

The natural setting for the study of multiresolutions and wavelet bases on Banach spaces appears to be the decomposition of the multiresolution subspaces by projection operators. A bounded linear operator $P$ on a Banach space $X$ is a projection if $P^{2}=P$. Associated with $P$ is the direct sum decomposition $X=V \oplus W$, where

$$
V:=P X \text { and } W:=\operatorname{Ker}(P),
$$

and $V \cap W=\{0\}$.

Let $\left\{V_{k}\right\}_{k \geq 0}$ be a multiresolution of $X_{2 \pi}$. For $k=0,1, \ldots$, let $P_{k}: X_{2 \pi} \rightarrow V_{k}$ be a projection onto $V_{k}$ and let

$$
W_{k}:=\operatorname{Ker}\left(\left.P_{k}\right|_{V_{k+1}}\right)
$$

Then

$$
V_{k+1}=V_{k} \oplus W_{k}, \quad V_{k} \cap W_{k}=\{0\} .
$$

The main object of this paper is the characterization of projections $P_{k}, k \geq 0$, so that $W_{k}$ have wavelet bases and constitute a Schauder decomposition of $X_{2 \pi}$. It unifies the study of wavelet decompositions by orthogonal projections in the Hilbert space $L_{2 \pi}^{2}$ on one hand and by interpolatory projections in the Banach space $C_{2 \pi}$ on the other. The approach is constructive and leads to the construction of a Schauder decomposition of $X_{2 \pi}$ and a biorthogonal system for $X_{2 \pi}$ and its dual $X_{2 \pi}^{*}$. We begin in Section 2 by studying the shift-invariant subspaces $V_{k}$ in the more general setting of a homogeneous Banach space $B$ on T. It is shown that $U_{k \geq 0} V_{k}$ is dense in $B$ if and only if $\left\{n \in \mathbf{Z}: \hat{\phi}_{k}(n)=0, k \geq 0\right\}=\left\{n \in \mathbf{Z}: e_{n} \notin B\right\}$, where $e_{n}(x)=e^{i n x}$. Section 3 gives a brief description of the construction of scaling functions $\phi_{k}$ and the corresponding basis $\left\{v_{k, j}: j=0,1, \ldots, 2^{k}-1\right\}$ for $V_{k}$. 
The projection $P_{k}$ is represented by a sequence of bounded linear functionals $v_{k, j}^{*} \in X_{2 \pi}^{*}, j=0,1, \ldots, 2^{k}-1$, viz.

$$
P_{k} f=\sum_{j=0}^{2^{k}-1}\left\langle f, v_{k, j}^{*}\right\rangle v_{k, j}, \quad f \in X_{2 \pi},
$$

where $\left\{v_{k, j}, v_{k, j}^{*}: j=0,1, \ldots, 2^{k}-1\right\}$ is a biorthogonal system, i.e.

$$
\left\langle v_{k, j}, v_{k, \ell}^{*}\right\rangle=\delta_{j, \ell}, \quad j, \ell=0,1, \ldots, 2^{k}-1
$$

Under the assumption that

$$
P_{k} T_{k}=T_{k} P_{k}
$$

the functionals $v_{k, j}^{*}, j=0,1, \ldots, 2^{k}-1$, and the adjoint shift operators, $T_{k}^{*}=T_{k}^{-1}$, enjoy the same properties as $v_{k, j}$ and $T_{k}$. These are studied in Section 4 where it is also shown that if $\left\{P_{k}\right\}_{k \geq 0}$ is uniformly bounded and satisfies

$$
P_{k} P_{k+1}=P_{k}, k=0,1, \ldots,
$$

then $\left\{V_{0}, W_{k}\right\}_{k \geq 0}$ is a Schauder decomposition of $X_{2 \pi}$. In Section 5 it is shown that a necessary and sufficient condition for $W_{k}$ to have a basis consisting of $\frac{2 \pi}{2^{k}}$-shifts of a function $\psi_{k}$ is that

$$
P_{k}\left(v_{k+1, j}\right)=\left\langle v_{k+1, j}, v_{k, j}^{*}\right\rangle v_{k, j}
$$

The dual subspaces

$$
V_{k}^{*}:=\operatorname{span}\left\{v_{k, j}^{*}: j=0,1, \ldots, 2^{k}-1\right\}, \quad k=0,1, \ldots,
$$

and the associated adjoint projections $P_{k}^{*}$ are studied in Section 6. It is shown that if $\left\{P_{k}\right\}_{k \geq 0}$ is uniformly bounded and satisfies (1.2), (1.3) and (1.4), then $\left\{V_{k}^{*}\right\}_{k \geq 0}$ is a multiresolution of $\bar{U}_{k \geq 0} V_{k}^{*}$. Further, if $X_{2 \pi}=L_{2 \pi}^{p}$ for $1<p<\infty$, then $U_{k \geq 0} V_{k}^{*}=L_{2 \pi}^{q}$ where $1 / p+1 / q=1$. A judicious approach in the construction of the wavelets $\left\{\psi_{k}\right\}_{k \geq 0}$ and $\left\{\psi_{k}^{*}\right\}_{k \geq 0}$ from the multiresolutions $\left\{V_{k}\right\}_{k \geq 0}$ and $\left\{V_{k}^{*}\right\}_{k \geq 0}$ respectively, gives a biorthogonal system

$$
\left\{\left\{\phi_{0}, T_{k}^{\ell} \psi_{k}\right\},\left\{\phi_{0}^{*}, T_{k}^{\ell} \psi_{k}^{*}\right\}: k=0,1, \ldots, \ell=0,1, \ldots, 2^{k}-1\right\}
$$

The Schauder decomposition of $X_{2 \pi}$ and the bases for $V_{k}$ and $W_{k}$ lead to decomposition and reconstruction algorithms. They are derived in Section 7. The paper concludes with two examples, one is on multiresolutions of $L_{2 \pi}^{2}$ in which the Schauder decomposition is accomplished by the least squares approximation operators, and the 
other is on multiresolutions of $C_{2 \pi}$ in which the decomposition is achieved by the interpolatory projections.

\section{Shift-invariant subspaces in $B$}

Let $B$ be a homogeneous Banach space on $\mathbf{T}$. Let $S$ be a finite-dimensional linear subspace of $B$ and suppose that there is a function $\phi \in B$ such that

$$
S=\left\{f \in B: f=\frac{1}{\sqrt{2^{k}}} \sum_{j=0}^{2^{k}-1} \alpha(j) T_{k}^{j} \phi, \alpha \in \mathcal{S}\left(2^{k}\right)\right\}
$$

where $S(N)$ denotes the class of all complex-valued periodic sequences of period $N$ equipped with the norm

$$
\|\alpha\|:=\left(\sum_{j=0}^{N-1}|\alpha(j)|^{2}\right)^{\frac{1}{2}}, \quad \alpha \in \mathcal{S}(N)
$$

Because of the equivalent representations

$$
f=\frac{1}{\sqrt{2^{k}}} \sum_{j=0}^{2^{k}-1} \alpha(j) T_{k}^{j} \phi \Longleftrightarrow \hat{f}(n)=\hat{\alpha}(n) \hat{\phi}(n), \text { for all } n \in \mathbf{Z}
$$

where

$$
\hat{\alpha}(n):=\frac{1}{\sqrt{2^{k}}} \sum_{j=0}^{2^{k}-1} \alpha(j) \omega_{k}^{-n j}, \quad \omega_{k}:=e^{\frac{p^{k}}{2^{k}}}
$$

$S$ can be expressed in the alternative form

$$
S=\left\{f \in B: \hat{f}(n)=\hat{\alpha}(n) \hat{\phi}(n), \quad \hat{\alpha} \in \mathcal{S}\left(2^{k}\right)\right\}
$$

The following result gives some equivalent conditions for MR1.

Proposition 2.1. Let $\phi \in L_{2 \pi}^{1}$, and

$$
v_{j}:=\frac{1}{\sqrt{2^{k}}} \sum_{\ell=0}^{2^{k}-1} \omega_{k}^{j \ell} T_{k}^{\ell} \phi, j=0,1, \ldots, 2^{k}-1 .
$$

Then the following are equivalent: 
(i) $\left\{T_{k}^{\ell} \phi: \ell=0,1, \ldots, 2^{k}-1\right\}$ is linearly independent.

(ii) $\left\{v_{j}: j=0,1, \ldots, 2^{k}-1\right\}$ is linearly independent.

(iii) $v_{j} \neq 0$ for $j=0,1, \ldots, 2^{k}-1$.

(iv) For each $j=0,1, \ldots, 2^{k}-1$,

$$
\hat{\phi}\left(j+2^{k} p\right) \neq 0
$$

for some $p \in \mathbf{Z}$.

Proof. The equivalence of (i) and (ii), and the implication (ii) $\Rightarrow$ (iii), are obvious. The equivalence of (iii) and (iv) follows from the observation that for $j=0,1, \ldots, 2^{k}-1$,

$$
v_{j}(x) \sim \sqrt{2^{k}} \sum_{p \in \mathbf{Z}} \hat{\phi}\left(j+2^{k} p\right) e^{\left(U+2^{k} p\right) x}
$$

where the notation $\sim$ means that the expression on the right-hand side is the Fourier series of the function on the left-hand side.

Suppose that (iv) holds, and let

$$
f:=\frac{1}{\sqrt{2^{k}}} \sum_{j=0}^{2^{k}-1} \alpha(j) T_{k}^{j} \phi
$$

be 0 . By (2.1),

$$
\hat{\alpha}(n) \hat{\phi}(n)=\hat{f}(n)=0, \quad \text { for all } n \in \mathbf{Z} .
$$

For each $j=0,1, \ldots, 2^{k}-1$, since $\hat{\phi}\left(j+2^{k} p\right) \neq 0$ for some $p \in \mathbf{Z}, \hat{\alpha}(j)=\hat{\alpha}\left(j+2^{k} p\right)=0$. Therefore $\alpha(j)=0$ for $j=0,1, \ldots, 2^{k}-1$, and (i) holds.

We now consider a sequence $\left\{V_{k}\right\}_{k \geq 0}$ of closed linear subspaces of $B$ and give a necessary and sufficient condition for $\left\{V_{k}\right\}_{k \geq 0}$ to satisfy the multiresolution condition MR3 if it satisfies MR2. For $n \in Z$, let $e_{n}(x)=e^{i n x}$.

Theorem 2.1. Let $\phi_{k} \in B, k=0,1, \ldots$, and $V_{k}=\operatorname{span}\left\{T_{k}^{\ell} \phi_{k}: \ell=0,1, \ldots, 2^{k}-1\right\}$. If $\left\{V_{k}\right\}_{k \geq 0}$ satisfies the condition MR2, then $U_{k \geq 0} V_{k}$ is dense in $B$ if and only if the set $\left\{n \in \mathbf{Z}: \hat{\phi}_{k}(n)=0\right.$, for all $\left.k \geq 0\right\}$ equals $\left\{n \in \mathbf{Z}: e_{n} \notin B\right\}$.

Corollary 2.1. Let $\phi_{k} \in X_{2 x}, k=0,1, \ldots$, and $V_{k}=\operatorname{span}\left\{T_{k}^{\ell} \phi_{k}: \ell=0,1, \ldots, 2^{k}-1\right\}$. If $\left\{V_{k}\right\}_{k \geq 0}$ satisfies the condition MR2, then $\cup_{k \geq 0} V_{k}$ is dense in $X_{2 x}$ if and only if the set $\left\{n \in Z: \hat{\phi}_{k}(n)=0\right.$, for all $\left.k \geq 0\right\}$ is empty. 
In order to prove the theorem, we first establish some auxiliary results. A subset $S$ of $B$ is translation invariant if $f \in S \Longrightarrow T_{t} f:=f(-t) \in S$ for all $t \in[0,2 \pi)$. We remark that $T_{t}, t \in[0,2 \pi)$, is a strongly continuous periodic semi-group of operators on $B$ and that for each $f \in B$, the map $\mathbf{R} \rightarrow B$ such that $t \rightarrow T_{\imath} f$ is continuous.

Lemma 2.1. Let $V$ be a subset of $B$. If $V$ satisfies the condition

$$
f \in V \Longrightarrow f\left(-\frac{2 \pi}{2^{k}}\right) \in V, \quad \text { for all } k=0,1, \ldots,
$$

then its closure $\bar{V}$ is translation invariant.

Proof. Suppose that $V$ satisfies (2.3). For any positive integer $n$, let

$$
t_{n}:=2 \pi\left\{\frac{a_{1}}{2}+\frac{a_{2}}{2^{2}}+\cdots+\frac{a_{n}}{2^{n}}\right\}
$$

where $a_{j} \in\{0,1\}$. Then

$$
T_{t_{n}} f=T_{1}^{a_{1}} T_{2}^{a_{2}} \cdots T_{n}^{a_{n}} f
$$

Therefore, $f \in V \Longrightarrow T_{t_{n}} f \in V$.

Now for any $t \in[0,2 \pi)$, there is a sequence $\left\{t_{n}\right\}$ with terms of the form (2.4) converging to $t$. It follows that $f \in V \Longrightarrow T_{\imath} f \in \bar{V}$. If $f \in \bar{V}$ and $\left\{f_{n}\right\}$ is a sequence in $V$ converging to $f$, then $T_{t} f_{n} \in \bar{V}$ for all $n$, and since $T_{t}$ is a bounded linear operator on $B$, it follows that $T_{t} f \in \bar{V}$.

For a subset $S$ of $B$, let $Z(S)=\cap_{f \in S}\{n \in Z: \hat{f}(n)=0\}$. The next lemma is a known result in harmonic analysis $([8$, p. 17 , Exercise 14$])$. For the sake of completeness, we include here a sketch of its proof.

Lemma 2.2. Let $H$ be a closed translation invariant linear subspace of $B$. Then

$$
Z(H)=\left\{n \in \mathbf{Z}: e_{n} \notin H\right\},
$$

and

$$
H=\overline{\operatorname{span}}\left\{e_{n}: n \notin Z(H)\right\}
$$

Proof. We first remark that if $f \in H$ and $g \in L_{2 \pi}^{1}$, then the usual convolution $f * g$ of $f$ and $g$ as $L^{1}$-functions is an element of $H$. The proof of this fact follows from a slight modification of an argument in [15, p. 128], and will be omitted here.

If $n \notin Z(H)$, then $\hat{f}(n) \neq 0$ for some $f$ in $H$. Since $\hat{f}(n) e_{n}=f * e_{n}$, by the above remark, 
$e_{n} \in H$. Conversely, if $e_{n} \in H$, then since $\hat{e}_{n}(n)=1$, we have $n \notin Z(H)$. Therefore (2.5) holds.

Let $f \in H$. For $n=1,2, \ldots$, let

$$
\sigma_{n}\left(f(x):=\sum_{j=-n}^{n}\left(1-\frac{|j|}{n+1}\right) \hat{f}(j) e^{i j x} .\right.
$$

If $\hat{f}(j) \neq 0$, then $j \notin Z(H)$. Hence $\sigma_{n}(f) \in \operatorname{span}\left\{e_{j}: j \notin Z(H)\right\}$. Since $\left\|\sigma_{n}(f)-f\right\|_{B} \rightarrow 0$ as $n \rightarrow \infty$ (see [8, p. 15]), $f \in \overline{\operatorname{span}}\left\{e_{j}: j \notin Z(H)\right\}$, and one direction of the inclusion in (2.6) is proved. By (2.5), the reverse inclusion in (2.6) is obvious.

Lemma 2.3. Let $V$ be a linear subspace of $B$ satisfying (2.3). Then $V$ is dense in $B$ if and only if

$$
Z(V)=\left\{n \in Z: e_{n} \notin B\right\}
$$

Proof. By Lemma 2.1, $\bar{V}$ is translation invariant. Applying Lemma 2.2 to $\bar{V}$ and $B, \bar{V}=B$ if and only if $Z(\bar{V})=Z(B)$, which is equivalent to (2.7).

Proof of Theorem 2.1. Let $V:=\cup_{k \geq 0} V_{k}$. Then $V$ satisfies (2.3), and by Lemma 2.3, it suffices to note that

$$
\bigcap_{f \in V}\{n \in \mathbf{Z}: \hat{f}(n)=0\}=\left\{n \in \mathbf{Z}: \hat{\phi}_{k}(n)=0, \quad \text { for all } k \geq 0\right\}
$$

which follows from the representation

$$
V_{k}=\left\{f \in B: \hat{f}(n)=\hat{\alpha}(n) \hat{\phi}_{k}(n), \hat{\alpha} \in \mathcal{S}\left(2^{k}\right)\right\}, \quad k=0,1, \ldots
$$

\section{Construction of scaling functions}

The idea in the construction of scaling functions which generate a multiresolution of $X_{2 \pi}$ is the same as that in [9]. We shall omit these details which can be found there.

Our starting point is a family of periodic sequences $\hat{h}_{k} \in \mathcal{S}\left(2^{k}\right), k \geq 1$, satisfying the following conditions:

$$
1-\hat{h}_{k}(n)=O\left(k^{-1-q}\right) \text { as } k \rightarrow \infty
$$

for some $\epsilon>0$ and for all $n \in Z$, and 


$$
\left|\hat{h}_{k+1}(j)\right|+\left|\hat{h}_{k+1}\left(j+2^{k}\right)\right| \leq 1, \quad j=0,1, \ldots, 2^{k}-1 .
$$

Let

$$
a_{k}(n):=\prod_{\ell=k+1}^{\infty} \hat{h}_{\ell}(n), \quad n \in \mathbf{Z} .
$$

Condition (3.1) implies that if $\hat{h}_{\ell}(n) \neq 0$ for $\ell \geq k+1$, then the infinite product in (3.3) converges to a nonzero number. In fact, for a fixed $n, \hat{h}_{\ell}(n)>0$ for all sufficiently large $\ell$. Hence $a_{k}(n)>0$ for all sufficiently large $k$. Because of this, it is reasonable to further assume that for $k \geq 0$,

$$
\hat{h}_{\ell}(n)>0 \text { for all } \ell \geq k+1 \text {, and for all }|n| \leq 2^{k-1} .
$$

This implies that

$$
a_{k}(n)>0 \text { for all }|n| \leq 2^{k-1}
$$

Theorem 3.1. If $\hat{h}_{k+1}$ satisfy (3.1), (3.2) and (3.4) for $k \geq 0$, then for $n \in Z$,

$$
\begin{gathered}
a_{k}(n) \rightarrow 1, \quad k \rightarrow \infty, \\
a_{k}(n)=\hat{h}_{k+1}(n) a_{k+1}(n), \quad k \geq 0,
\end{gathered}
$$

and for $j=0,1, \ldots, 2^{k}-1$,

$$
0<\sum_{p \in \mathbf{Z}}\left|a_{k}\left(j+2^{k} p\right)\right| \leq 1
$$

Proof. Condition (3.1) implies that for each $n \in Z$, there is an integer $m$ such that $a_{m}(n) \neq 0$. Writing

$$
a_{m}(n)=\left(\prod_{\ell=m+1}^{k} \hat{h}_{\ell}(n)\right) a_{k}(n)
$$

and taking limit as $k \rightarrow \infty$ leads to (3.6). The relation (3.7) follows directly from (3.3).

The left inequality in (3.8) follows from (3.4) and (3.5). The inequality on the right of (3.8) follows by a similar argument as in [9]. 
Corollary 3.1. For $k \geq 0$, let $\hat{h}_{k+1} \in \mathcal{S}\left(2^{k+1}\right)$ satisfy (3.1), (3.2) and (3.4), and suppose that $a_{k}$ are defined in (3.3). Define $\phi_{k} \in X_{2 \pi}$ such that $\hat{\phi}_{k}(n):=a_{k}(n), n \in \mathbf{Z}$. Then $\hat{\phi}_{k} \in \ell^{1}(\mathbf{Z})$,

$$
\phi_{k}(x):=\sum_{n \in \mathbf{Z}} \hat{\phi}_{k}(n) e^{i n x}, \text { uniformly in } \mathbf{R}
$$

and $\phi_{k}, k \geq 0$, generate a multiresolution of $X_{2 \pi}$.

\section{Multiresolution and projections on $X_{2 \pi}$}

Let $X$ be a Banach space and $X^{*}$ denote its dual. Associated with a projection operator $P: X \rightarrow X$ is the direct sum decomposition $X=V \oplus W$, where

$$
V:=P X, \quad W:=\operatorname{Ker}(P) .
$$

We shall also call $P$ a projection on $V$. If $V$ is a finite-dimensional subspace of $X$ and $\left\{v_{1}, v_{2}, \ldots, v_{n}\right\}$ is a basis for $V$, then $P$ can be expressed as

$$
P f=\sum_{j=1}^{n}\left\langle f, v_{j}^{*}\right\rangle v_{j}, \quad f \in X,
$$

where $v_{j}^{*}: X \rightarrow \mathbf{C}, j=1,2, \ldots, n$, are bounded linear functionals satisfying

$$
\begin{aligned}
\left\langle v_{\ell}, v_{j}^{*}\right\rangle & =\delta_{j, \ell}, \quad j, \ell=1,2, \ldots, n, \\
\left\langle g, v_{j}^{*}\right\rangle & =0 \text { for all } g \in \operatorname{Ker}(P) .
\end{aligned}
$$

Here, $\langle$,$\rangle denotes the dual action of the functionals, i.e.$

$$
\left\langle f, g^{*}\right\rangle:=g^{*}(f) \text { or }\left\langle f, g^{*}\right\rangle:=J(f)\left(g^{*}\right), \quad f \in X, g^{*} \in X^{*},
$$

where $J: X \rightarrow X^{* *}$ is the natural embedding of $X$ into $X^{* *}$.

Suppose that $\left\{V_{k}\right\}_{k \geq 0}$ is a multiresolution of $X_{2 \pi}$ generated by $\phi_{k}, k \geq 0$. Condition MR2 gives the scaling relation

$$
\phi_{k}=\frac{1}{\sqrt{2^{k+1}}} \sum_{\ell=0}^{2^{k+1}-1} h_{k+1}(\ell) T_{k+1}^{\ell} \phi_{k+1}
$$

which is equivalent to

$$
\hat{\phi}_{k}(n)=\hat{h}_{k+1}(n) \hat{\phi}_{k+1}(n), \quad n \in \mathbf{Z}
$$


where

$$
h_{k+1}(\ell)=\frac{1}{\sqrt{2^{k+1}}} \sum_{n=0}^{2^{k+1}-1} \hat{h}_{k+1}(n) \omega_{k+1}^{\ell n}
$$

and

$$
\hat{h}_{k+1}(n):=\frac{1}{\sqrt{2^{k+1}}} \sum_{\ell=0}^{2^{k+1}-1} h_{k+1}(\ell) \omega_{k+1}^{-n \ell} .
$$

The sequence $\hat{h}_{k+1}$ is the finite Fourier transform of $h_{k+1}$.

For $k=0,1, \ldots$, and $j=0,1, \ldots, 2^{k}-1$, as in Proposition 2.1 , we define

$$
v_{k, j}:=\frac{1}{\sqrt{2^{k}}} \sum_{\ell=0}^{2^{k}-1} \omega_{k}^{j \ell} T_{k}^{\ell} \phi_{k} .
$$

Then

$$
T_{k}^{\ell} \phi_{k}=\frac{1}{\sqrt{2^{k}}} \sum_{j=0}^{2^{k}-1} \omega_{k}^{-\ell j} v_{k, j}
$$

and

$$
T_{k}^{\ell} v_{k, j}=\omega_{k}^{-\ell j} v_{k, j}
$$

Remark 1. Proposition 2.1 shows that $\left\{v_{k, j}: j=0,1, \ldots, 2^{k}-1\right\}$ is linearly independent.

It follows from (4.3) that for $k=0,1, \ldots$, and $j=0,1, \ldots, 2^{k}-1$,

$$
v_{k, j}(x) \sim \sqrt{2^{k}} \sum_{p \in Z} \hat{\phi}_{k}\left(j+2^{k} p\right) e^{i\left(j+2^{k} p\right) x}
$$

Equating Fourier coefficients, (4.6) and (4.2) lead to

$$
v_{k, j}=\frac{1}{\sqrt{2}} \hat{h}_{k+1}(j) v_{k+1, j}+\frac{1}{\sqrt{2}} \hat{h}_{k+1}\left(j+2^{k}\right) v_{k+1, j+2^{k}} .
$$

More generally if $f \in V_{k+1}$, then 


$$
f(x)=\frac{1}{\sqrt{2^{k+1}}} \sum_{\ell=0}^{2^{k+1}-1} \alpha(\ell) T_{k+1}^{\ell} \phi_{k+1}(x),
$$

and its Fourier coefficients $\hat{f}(n)$ satisfy

$$
\hat{f}(n)=\hat{\alpha}(n) \hat{\phi}_{k+1}(n), \quad n \in \mathbf{Z},
$$

where $\hat{\alpha} \in \mathcal{S}\left(2^{k+1}\right)$. As before, if we define

$$
f_{j}:=\frac{1}{\sqrt{2^{k}}} \sum_{\ell=0}^{2^{k}-1} \omega_{k}^{j \ell} T_{k}^{\ell} f, \quad j=0,1, \ldots, 2^{k}-1
$$

then

$$
\begin{gathered}
T_{k}^{\ell} f=\frac{1}{\sqrt{2^{k}}} \sum_{j=0}^{2^{k}-1} \omega_{k}^{-\ell j} f_{j}, \quad \ell=0,1, \ldots, 2^{k}-1, \\
f_{j}(x) \sim \sqrt{2^{k}} \sum_{p \in Z} \hat{f}\left(j+2^{k} p\right) e^{\ell\left(j+2^{k} p\right) x}
\end{gathered}
$$

and

$$
f_{j}=\frac{1}{\sqrt{2}} \hat{\alpha}(j) v_{k+1, j}+\frac{1}{\sqrt{2}} \hat{\alpha}\left(j+2^{k}\right) v_{k+1, j+2^{k}}
$$

for $j=0,1, \ldots, 2^{k}-1$.

Proposition 4.1. The following are equivalent:

(i) $\left\{T_{k}^{\ell} f: \ell=0,1, \ldots, 2^{k}-1\right\}$ is linearly independent.

(ii) $\left\{f_{j}: j=0,1, \ldots, 2^{k}-1\right\}$ is linearly independent.

(iii) For $j=0,1, \ldots, 2^{k}-1$,

$$
|\hat{\alpha}(j)|+\left|\hat{\alpha}\left(j+2^{k}\right)\right| \neq 0 .
$$

Proof. This follows from Proposition 2.1, (4.11) and the linear independence of the set $\left\{v_{k+1, j}, j=0,1, \ldots, 2^{k+1}-1\right\}$.

For $k=0,1, \ldots$, let $P_{k}: X_{2 \pi} \rightarrow V_{k}$ be a projection onto $V_{k}$. The multiresolution condition MR2 is equivalent to $P_{m} P_{k}=P_{k}$ for all $k \leq m$. For $k=0,1, \ldots$, 


$$
X_{2 \pi}=V_{k} \oplus \operatorname{Ker}\left(P_{k}\right) \text {. }
$$

We note that for a given sequence of projections $P_{k}, k=0,1, \ldots$, there are $v_{k, j}^{*} \in X_{2 \pi}^{*}, k=0,1, \ldots, j=0,1, \ldots, 2^{k}-1$, satisfying

$$
\left\langle v_{k, \ell}, v_{k, j}^{*}\right\rangle=\delta_{j, \ell}, \quad j, \ell=0,1, \ldots, 2^{k}-1,
$$

and

$$
\left(f, v_{k, j}^{*}\right)=0, \quad \text { for all } f \in \operatorname{Ker}\left(P_{k}\right)
$$

such that

$$
P_{k} f=\sum_{j=0}^{2^{k}-1}\left\langle f, v_{k, j}^{*}\right\rangle v_{k, j}
$$

On the other hand, given $v_{k, j}^{*} \in X_{2 \pi}^{*}, j=0,1, \ldots, 2^{k}-1$, satisfying (4.13), the mapping

$$
P_{k} f:=\sum_{j=0}^{2^{k}-1}\left\langle f, v_{k, j}^{*}\right) v_{k, j}, \quad f \in X_{2 \pi},
$$

is a projection from $X_{2 \pi}$ onto $V_{k}$ with $\operatorname{Ker}\left(P_{k}\right)=\cap_{j=0}^{2^{k}-1} \operatorname{Ker}\left(v_{k, j}^{*}\right)$.

Let $T_{k}^{*}: X_{2 \pi}^{*} \rightarrow X_{2 \pi}^{*}$ denote the adjoint of the shift operator $T_{k}$, and let

$$
\phi_{k}^{*}:=\frac{1}{\sqrt{2^{k}}} \sum_{j=0}^{2^{k}-1} v_{k, j}^{*}
$$

Note that $T_{k}^{*}=T_{k}^{-1}: X_{2 \pi}^{*} \rightarrow X_{2 \pi}^{*}$.

Proposition 4.2. For $j, \ell=0,1, \ldots, 2^{k}-1$,

$$
\hat{h}_{k+1}(j)\left\langle v_{k+1, j}, v_{k, \ell}^{*}\right\rangle+\hat{h}_{k+1}\left(j+2^{k}\right)\left(v_{k+1, j+2^{k}}, v_{k, \ell}^{*}\right)=\sqrt{2} \delta_{j, \ell},
$$

and

$$
\left\langle T_{k}^{\ell} \phi_{k}, T_{k}^{j} \phi_{k}^{*}\right\rangle=\delta_{j, \ell}
$$

Further, if

$$
P_{k} T_{k}=T_{k} P_{k}
$$

then 


$$
\begin{aligned}
& T_{k}^{\ell} \phi_{k}^{*}=\frac{1}{\sqrt{2^{k}}} \sum_{j=0}^{2^{k}-1} \omega_{k}^{\ell j} v_{k, j}^{*}, \\
& v_{k, j}^{*}=\frac{1}{\sqrt{2^{k}}} \sum_{\ell=0}^{2^{k}-1} \omega_{k}^{-j \ell} T_{k}^{\ell} \phi_{k}^{*},
\end{aligned}
$$

and

$$
\hat{v}_{k, j}^{*}(n)= \begin{cases}\sqrt{2^{k}} \hat{\phi}_{k}^{*}\left(-j+2^{k} p\right), & n=-j+2^{k} p, p \in \mathbf{Z}, \\ 0 & \text { otherwise. }\end{cases}
$$

Proof. The equation (4.17) follows from (4.7) and (4.13). The relations (4.4) and (4.13) imply that for $\ell, m=0,1, \ldots, 2^{k}-1$,

$$
\left\langle T_{k}^{\ell} \phi_{k}, v_{k, m}^{*}\right\rangle=\frac{1}{\sqrt{2^{k}}} \omega_{k}^{-\ell m} .
$$

Then (4.23) and the definition (4.16) give

$$
\left\langle T_{k}^{\ell} \phi_{k}, \phi_{k}^{*}\right\rangle=\frac{1}{2^{k}} \sum_{j=0}^{2^{k}-1} \omega_{k}^{-j \ell}=\delta_{0, \ell}
$$

which is equivalent to (4.18).

To prove $(4.20)$, observe that $(4.19)$ is equivalent to

$$
T_{k}^{\ell} v_{k, J}^{*}=w_{k}^{\ell j} v_{k, j}^{*}, \quad j, \ell=0,1, \ldots, 2^{k}-1,
$$

which together with (4.16) leads to (4.20). The relation (4.21) is the inverse finite Fourier transform of (4.20), and (4.22) follows from (4.21) by evaluating the Fourier coefficients.

Proposition 4.3. The following are equivalent:

(i) $P_{k} P_{k+1}=P_{k}, \quad k=0,1, \ldots$

(ii) $P_{k} P_{m}=P_{k}$ for $k \leq m$.

(iii) $\operatorname{Ker}\left(P_{m}\right) \subset \operatorname{Ker}\left(P_{k}\right)$ for $k \leq m$.

(iv) $v_{k, j}^{*}=\sum_{\ell=0}^{2^{k+1}-1}\left(v_{k+1, \ell}, v_{k, j}^{*}\right) v_{k+1, \ell}^{*}$ 
Proof. Obviously, (ii) implies (i). Conversely, if (i) holds, then for $k \leq m$,

$$
P_{k} P_{m}=P_{k} P_{k+1} P_{m}=\cdots=P_{k} P_{k+1} \cdots P_{m-1} P_{m}=P_{k} P_{k+1} \cdots P_{m-1}=\cdots=P_{k}
$$

Now $\operatorname{Ker}\left(P_{k}\right)$ is the image of the projection $I-P_{k}$. Hence (iii) holds if and only if $\left(I-P_{k}\right)\left(I-P_{m}\right)=I-P_{m}$, which is equivalent to (ii).

Finally, the relation (i) is equivalent to

$$
\sum_{j=0}^{2^{k}-1}\left\langle P_{k+1} f, v_{k, j}^{*}\right) v_{k, I}=\sum_{j=0}^{2^{k}-1}\left(f, v_{k, j}^{*}\right) v_{k, j}, \quad f \in X_{2 \pi},
$$

which is equivalent to (iv) since

$$
\left\langle P_{k+1} f, v_{k, j}^{*}\right\rangle=\sum_{\ell=0}^{2^{k+1}-1}\left\langle v_{k+1, \ell}, v_{k, j}^{*}\right\rangle\left\langle f, v_{k+1, \ell}^{*}\right\rangle, \quad f \in X_{2 \pi} .
$$

For each $k=0,1, \ldots,\left.P_{k}\right|_{V_{k+1}}$ is also a projection on $V_{k}$. Let

$$
W_{k}:=\operatorname{Ker}\left(\left.P_{k}\right|_{v_{k+1}}\right)=V_{k+1} \cap \operatorname{Ker}\left(P_{k}\right)
$$

Then

$$
V_{k+1}=V_{k} \oplus W_{k}, \quad k=0,1, \ldots
$$

If $\left\{V_{k}\right\}_{k \geq 0}$ is a multiresolution of $L_{2 \pi}^{2}$ and $P_{k}$ are orthogonal projections, then

$$
L_{2 \pi}^{2}=V_{0} \oplus^{\perp} W_{0} \oplus^{\perp} W_{1} \oplus^{\perp} \cdots
$$

The corresponding decomposition for $X_{2 \pi}$ is not true in general. If every $f \in X_{2 \pi}$ has a representation of the form

$$
f=f_{0}+\sum_{k=0}^{\infty} g_{k}
$$

where $f_{0} \in V_{0}, g_{k} \in W_{k}, k=0,1, \ldots$, and the series converges in the norm of $X_{2 \pi}$, then we shall express this by writing

$$
X_{2 \pi}=V_{0}+W_{0}+W_{1}+\cdots
$$

Further, if every $f \in X_{2 \pi}$ has a unique representation of the form (4.25), then $\left\{V_{0}, W_{k}\right\}_{k \geq 0}$ will be called a Schauder decomposition of $X_{2 \pi}$. In this case we shall write

$$
X_{2 \pi}=V_{0} \oplus W_{0} \oplus W_{1} \oplus \cdots
$$


The following result may be deduced from the theory of bases in Banach spaces (see [11]), but we shall give a direct proof for completeness.

Proposition 4.4. Let $\left\{V_{k}\right\}_{k \geq 0}$ be a multiresolution of $X_{2 \pi}$ and $P_{k}: X_{2 \pi} \rightarrow V_{k}$, $k=0,1, \ldots$, be a sequence of projection operators on $V_{k}$. If

$$
\left\{P_{k} f\right\}_{k \geq 0} \text { is a bounded sequence in } X_{2 \pi} \text { for each } f \in X_{2 \pi}
$$

and

$$
P_{k} P_{k+1}=P_{k} \text { for all } k=0,1, \ldots,
$$

then (4.27) holds, i.e. $\left\{V_{0}, W_{k}\right\}_{k \geq 0}$ is a Schauder decomposition of $X_{2 \pi}$.

Proof. Suppose that (4.28) and (4.29) hold. By the Uniform Boundedness Principle, (4.28) entails that $\left\{P_{k}\right\}_{k \geq 0}$ is uniformly bounded. Suppose that $\left\|P_{k}\right\| \leq M$ for all $k \geq 0$. Let $f \in X_{2 \pi}$. Then for any $\epsilon>0$, there exists a function $\tilde{f} \in \cup_{k \geq 0} V_{k}$ satisfying

$$
\|\tilde{f}-f\|<\frac{\epsilon}{M+1}
$$

It follows that there is a positive integer $N$ such that $\tilde{f} \in V_{k}$ for all $k \geq N$. Hence for $k \geq N$,

$$
\begin{aligned}
\left\|P_{k} f-f\right\| & \leq\left\|P_{k} f-P_{k} \tilde{f}\right\|+\left\|P_{k} \tilde{f}-f\right\| \\
& \leq\left(\left\|P_{k}\right\|+1\right)\|\tilde{f}-f\|<\epsilon .
\end{aligned}
$$

Hence the sequence $\left\{P_{k} f\right\}$ converges to $f$, and

$$
f=P_{0} f+\sum_{k=0}^{\infty}\left(P_{k+1} f-P_{k} f\right) .
$$

For $k=0,1, \ldots$, let

$$
Q_{k}=P_{k+1}-P_{k}
$$

It follows from (4.29) and Proposition 4.3 that $Q_{k}$ is a projection,

$$
\begin{gathered}
Q_{k} Q_{j}=0, \quad k \neq j, \\
P_{k} Q_{m}=Q_{m} P_{k}=0, \quad m \geq k,
\end{gathered}
$$

and 


$$
Q_{k}\left(X_{2 \pi}\right)=W_{k}
$$

Therefore (4.26) holds.

To prove (4.27) it suffices to show that if

$$
f_{0}+\sum_{k=0}^{\infty} g_{k}=0
$$

where $f_{0} \in V_{0}$ and $g_{k} \in W_{k}, k=0,1, \ldots$, then $f_{0}=g_{k}=0$ for all $k=0,1, \ldots$. Indeed, by (4.32)-(4.34), applying the operators $P_{0}$ and $Q_{k}$ to the functions in (4.35) gives $f_{0}=0$ and $g_{k}=0, k=0,1, \ldots$.

\section{Characterization of projections for a wavelet basis}

Suppose that $\left\{V_{k}\right\}_{k \geq 0}$ is a multiresolution of $X_{2 \pi}$ generated by scaling functions $\phi_{k}$. Let $P_{k}: X_{2 \pi} \rightarrow V_{k}, k=0,1, \ldots$, be a sequence of projections on $V_{k}$ and $W_{k}:=V_{k+1} \cap \operatorname{Ker}\left(P_{k}\right)$, so that

$$
V_{k+1}=V_{k} \oplus W_{k}, \quad k=0,1, \ldots
$$

We shall characterize the projections $P_{k}, k=0,1, \ldots$, for which each $W_{k}$ has a basis generated by $\frac{2 \pi}{2^{k}}$-shifts of a function. The approach is constructive and leads to the construction of such a basis. Note that in the construction we do not assume $P_{k}$ satisfy (4.19), (4.28) and (4.29).

Let $\psi_{k} \in V_{k+1}$. Then

$$
\hat{g}_{k+1}(j) \hat{\phi}_{k+1}\left(j+2^{k+1} p\right)=\hat{\psi}_{k}\left(j+2^{k+1} p\right)
$$

for all $p \in \mathbf{Z}, j=0,1, \ldots, 2^{k+1}-1$, for some $\hat{g}_{k+1} \in \mathcal{S}\left(2^{k+1}\right)$. For $j=0,1, \ldots, 2^{k}-1$, let

$$
u_{k, j}:=\frac{1}{\sqrt{2^{k}}} \sum_{\ell=0}^{2^{k}-1} \omega_{k}^{\jmath \ell} T_{k}^{\ell} \psi_{k}
$$

Then by (4.9), (4.10) and (4.11),

$$
\begin{gathered}
T_{k}^{\ell} \psi_{k}=\frac{1}{\sqrt{2^{k}}} \sum_{j=0}^{2^{k}-1} \omega_{k}^{-\ell j} u_{k, j}, \quad \ell=0,1, \ldots, 2^{k}-1, \\
u_{k, j}(x) \sim \sqrt{2^{k}} \sum_{p \in \mathbf{Z}} \hat{\psi}_{k}\left(j+2^{k} p\right) e^{i\left(j+2^{k} p\right) x}
\end{gathered}
$$


and

$$
u_{k, j}=\frac{1}{\sqrt{2}} \hat{g}_{k+1}(j) v_{k+1, j}+\frac{1}{\sqrt{2}} \hat{g}_{k+1}\left(j+2^{k}\right) v_{k+1, j+2^{k}}
$$

for $j=0,1, \ldots, 2^{k}-1$.

Proposition 5.1. The functions $u_{k, j} \in W_{k}, j=0,1, \ldots, 2^{k}-1$, if and only if

$$
\hat{g}_{k+1}(j)\left\langle v_{k+1, j}, v_{k, \ell}^{*}\right)+\hat{g}_{k+1}\left(j+2^{k}\right)\left\langle v_{k+1, j+2^{k}}, v_{k, \ell}^{*}\right\rangle=0, \quad \ell, j=0,1, \ldots, 2^{k}-1 .
$$

Further, $\left\{T_{k}^{\ell} \psi_{k}: \ell=0,1, \ldots, 2^{k}-1\right\}$ is a basis for $W_{k}$ if and only if (5.6) holds and

$$
\left|\hat{g}_{k+1}(j)\right|+\left|\hat{g}_{k+1}\left(j+2^{k}\right)\right| \neq 0, \quad j=0,1, \ldots, 2^{k}-1 .
$$

Proof. Since $u_{k, j} \in V_{k+1}$, it follows that $u_{k, j} \in W_{k}$ if and only if $u_{k, j} \in \operatorname{Ker}\left(P_{k}\right) \Longleftrightarrow$ $\left\langle u_{k, j}, v_{k, \ell}^{*}\right\rangle=0$ for $\ell=0,1, \ldots, 2^{k}-1$, which is equivalent to (5.6).

The second assertion follows from Proposition 4.1.

Theorem 5.1. Suppose that $v_{k, j}^{*} \in X_{2 \pi}^{*}$ are the associated bounded linear functionals of the projection operators $P_{k}$. The following are equivalent:

(i) There exists a function $\psi_{k} \in V_{k+1}$ such that $\left\{T_{k}^{\ell} \psi_{k}: \ell=0,1, \ldots, 2^{k}-1\right\}$ is a basis for $W_{k}$.

(ii) For $j, \ell=0,1, \ldots, 2^{k}-1$,

$$
\left\langle v_{k+1, j}, v_{k, \ell}^{*}\right\rangle=\left\langle v_{k+1, j+2^{k}}, v_{k, \ell}^{*}\right\rangle=0, \quad j \neq \ell .
$$

(iii) For $j=0,1, \ldots, 2^{k+1}-1$,

$$
P_{k}\left(v_{k+1, f}\right)=\left(v_{k+1, j}, v_{k, j}^{*}\right) v_{k, j}
$$

where $v_{k, j}$ and $v_{k, j}^{*}$ are assumed to be periodic with respect to the second index $j$, with period $2^{k}$.

Proof. For convenience we let

$$
\beta_{j, \ell}^{k}:=\left\langle v_{k+1, j}, v_{k, \ell}^{*}\right\rangle, \quad j=0,1, \ldots, 2^{k+1}-1, \ell=0,1, \ldots, 2^{k}-1 .
$$

Suppose that (i) holds. Then there exists $\hat{g}_{k+1} \in \mathcal{S}\left(2^{k+1}\right)$ such that $\psi_{k}$ satisfies (5.1) and $\hat{g}_{k+1}$ satisfies (5.6) and (5.7), by Proposition 5.1. Hence at least one of $\hat{g}_{k+1}(j)$ and $\hat{g}_{k+1}\left(j+2^{k}\right)$ is nonzero. If (5.8) is false, then there exists $j \neq \ell$ such that at least one of $\beta_{j, \ell}^{k}$ and $\beta_{j+2^{k}, \ell}^{k}$ is nonzero. For these values of $j, \ell,(4.17)$ gives 


$$
\hat{h}_{k+1}(j) \beta_{j, \ell}^{k}+\hat{h}_{k+1}\left(j+2^{k}\right) \beta_{j+2^{k}, \ell}^{k}=0 .
$$

In addition (4.17) implies that

$$
\hat{h}_{k+1}(j) \beta_{j, j}^{k}+\hat{h}_{k+1}\left(j+2^{k}\right) \beta_{j+2^{k}, j}^{k}=\sqrt{2}
$$

Equations (5.11) and (5.12) form a linear system in $\left(\hat{h}_{k+1}(j), \hat{h}_{k+1}\left(j+2^{k}\right)\right)^{r}$ whose matrix is

$$
A=\left(\begin{array}{cc}
\beta_{j, J}^{k} & \beta_{j+2^{k} . J}^{k} \\
\beta_{j, \ell}^{k} & \beta_{j+2^{k}, \ell}^{k}
\end{array}\right) .
$$

The matrix $A$ is nonsingular. Otherwise,

$$
\left(\beta_{j, \ell}^{k}, \beta_{j+2^{k}, \ell}^{k}\right)=c\left(\beta_{j, j}^{k}, \beta_{j+2^{k}, j}^{k}\right),
$$

for some $c \neq 0$, but (5.11), (5.12) and (5.14) imply that $c=0$.

Now for these values of $j, \ell$, the $j$-th and $\ell$-th equations in (5.6) can be written as

$$
A\left(\begin{array}{c}
\hat{g}_{k+1}(j) \\
\hat{g}_{k+1}\left(j+2^{k}\right)
\end{array}\right)=0
$$

Hence $\hat{g}_{k+1}(j)=\hat{g}_{k+1}\left(j+2^{k}\right)=0$, which is a contradiction.

If (ii) holds, then (4.17) and (5.6) are equivalent to

$$
\begin{gathered}
\hat{h}_{k+1}(j) \beta_{j, j}^{k}+\hat{h}_{k+1}\left(j+2^{k}\right) \beta_{j+2^{k}, j}^{k}=\sqrt{2}, \\
\hat{g}_{k+1}(j) \beta_{j, j}^{k}+\hat{g}_{k+1}\left(j+2^{k}\right) \beta_{j+2^{k}, j}^{k}=0,
\end{gathered}
$$

for $j=0,1, \ldots, 2^{k}-1$. Given $\hat{h}_{k+1}, \beta_{j, j}^{k}$ and $\beta_{j+2^{k}, j}^{k}, j=0,1, \ldots, 2^{k}-1$, satisfying (5.15), we shall construct a periodic sequence $\hat{g}_{k+1} \in \mathcal{S}\left(2^{k+1}\right)$ satisfying (5.16) so that the $\frac{2 \pi}{2^{k}}$ shifts of the function $\psi_{k}$ whose Fourier sequence satisfies (5.1), form a basis for $W_{k}$. The construction proceeds as follows. For $j=0,1, \ldots, 2^{k}-1$,

(a) if $\beta_{j, j}^{k} \neq 0$, we let $\hat{g}_{k+1}\left(j+2^{k}\right)$ be an arbitrary nonzero number and set

$$
\hat{g}_{k+1}(j):=\frac{-\hat{g}_{k+1}\left(j+2^{k}\right) \beta_{j+2^{k}, j}^{k}}{\beta_{j, j}^{k}}
$$

(b) if $\beta_{j, j}^{k}=0$, we let $\hat{g}_{k+1}(j)$ be an arbitrary nonzero number and set $\hat{g}_{k+1}\left(j+2^{k}\right):=0$.

In either case, $\hat{g}_{k+1}$ satisfies (5.6) and (5.7), which implies (i) by Proposition 5.1.

The equivalence of (ii) and (iii) follows directly from the representation (4.15). 
Remark 2. If (ii) holds, steps (a) and (b) above give a construction of the sequence $\hat{g}_{k+1}$ and hence the wavelet $\psi_{k}$.

\section{Dual multiresolution and adjoint projections}

Suppose that $\left\{V_{k}\right\}_{k \geq 0}$ is a multiresolution of $X_{2 \pi}$ generated by the scaling functions $\phi_{k}$ and let $P_{k}: X_{2 \pi} \rightarrow V_{k}, k=0,1, \ldots$, be a sequence of projection operators on $V_{k}$. Henceforward, we shall assume that $P_{k}, k \geq 0$, satisfy (4.19), (4.28), (4.29) and (5.9). Because of (5.8) we define $\hat{h}_{k+1}^{*} \in \mathcal{S}\left(2^{k+1}\right)$, by

$$
\hat{h}_{k+1}^{*}(j):=\sqrt{2}\left(v_{k+1, j}, v_{k, j}^{*}\right), \quad j=0,1, \ldots, 2^{k+1}-1
$$

with the usual assumption that $v_{k, j}^{*}$ is periodic in $j$ of period $2^{k}$. In terms of $\beta_{j, \ell}^{k}$,

$$
\hat{h}_{k+1}^{*}(j)=\left\{\begin{array}{l}
\sqrt{2} \beta_{j, j}^{k}, \quad j=0,1, \ldots, 2^{k}-1, \\
\sqrt{2} \beta_{j, j-2^{k}}^{k}, \quad j=2^{k}, \ldots, 2^{k+1}-1 .
\end{array}\right.
$$

Let

$$
\left\{u_{k, j}: j=0,1, \ldots, 2^{k}-1\right\} \text { and }\left\{T_{k}^{\ell} \psi_{k}: \ell=0,1, \ldots, 2^{k}-1\right\}
$$

be the bases for $W_{k}$ constructed in Section 5 , where the function $\psi_{k}$ is defined via its Fourier coefficients by

$$
\hat{g}_{k+1}(j) \hat{\phi}_{k+1}\left(j+2^{k+1} p\right)=\hat{\psi}_{k}\left(j+2^{k+1} p\right)
$$

for all $p \in Z, j=0,1, \ldots, 2^{k+1}-1$,

$$
u_{k, j}:=\frac{1}{\sqrt{2^{k}}} \sum_{\ell=0}^{2^{k}-1} \omega_{k}^{\ell} T_{k}^{\ell} \psi_{k}
$$

equivalently,

$$
T_{k}^{\ell} \psi_{k}=\frac{1}{\sqrt{2^{k}}} \sum_{j=0}^{2^{k}-1} \omega_{k}^{-\ell j} u_{k, j}, \quad \ell=0,1, \ldots, 2^{k}-1
$$

and for $j=0,1, \ldots, 2^{k}-1$,

(6a) if $\hat{h}_{k+1}^{*}(j) \neq 0$, then $\hat{g}_{k+1}\left(j+2^{k}\right)$ is an arbitrary nonzero number and 


$$
\hat{g}_{k+1}(j):=\frac{-\hat{g}_{k+1}\left(j+2^{k}\right) \hat{h}_{k+1}^{*}\left(j+2^{k}\right)}{\hat{h}_{k+1}^{*}(j)}
$$

(6b) if $\hat{h}_{k+1}^{*}(j)=0$, then $\hat{g}_{k+1}(j)$ is an arbitrary nonzero number and $\hat{g}_{k+1}\left(j+2^{k}\right):=0$.

Proposition 6.1. Suppose that $P_{k}, k=0,1, \ldots$, satisfy (4.28) and (4.29). Then

$$
\operatorname{Ker}\left(P_{k}\right)=W_{k} \oplus W_{k+1} \oplus \cdots
$$

Proof. By Proposition 4.4,

$$
X_{2 \pi}=V_{k} \oplus W_{k} \oplus W_{k+1} \oplus \cdots
$$

We also have another direct sum decomposition

$$
X_{2 \pi}=V_{k} \oplus \operatorname{Ker}\left(P_{k}\right)
$$

By Proposition 4.3, $W_{m} \subset \operatorname{Ker}\left(P_{k}\right)$ for all $m \geq k$, and so

$$
W_{k} \oplus W_{k+1} \oplus \cdots \subset \operatorname{Ker}\left(P_{k}\right)
$$

This inclusion and the uniqueness of the above two decompositions of $X_{2 \pi}$ give the equality (6.7).

For $k=0,1, \ldots$, let

$$
V_{k}^{*}:=\operatorname{span}\left\{v_{k, J}^{*}: j=0,1, \ldots, 2^{k}-1\right\}
$$

Then (iv) of Proposition 4.3 implies that

$$
V_{k}^{*} \subset V_{k+1}^{*}, \quad k=0,1, \ldots
$$

Further, (5.8) and (iv) of Proposition 4.3 give

$$
v_{k, j}^{*}=\frac{1}{\sqrt{2}} \hat{h}_{k+1}^{*}(j) v_{k+1, j}^{*}+\frac{1}{\sqrt{2}} \hat{h}_{k+1}^{*}\left(j+2^{k}\right) v_{k+1, j+2^{k}}^{*},
$$

which is the dual of the relation (4.7):

$$
v_{k, j}=\frac{1}{\sqrt{2}} \hat{h}_{k+1}(j) v_{k+1, j}+\frac{1}{\sqrt{2}} \hat{h}_{k+1}\left(j+2^{k}\right) v_{k+1, j+2^{k}} .
$$

It is also convenient to write the equations $(5.15)$ and $(5.16)$ in the following forms 
which show the duality:

$$
\begin{aligned}
& \hat{h}_{k+1}(j) \hat{h}_{k+1}^{*}(j)+\hat{h}_{k+1}\left(j+2^{k}\right) \hat{h}_{k+1}^{*}\left(j+2^{k}\right)=2, \\
& \hat{g}_{k+1}(j) \hat{h}_{k+1}^{*}(j)+\hat{g}_{k+1}\left(j+2^{k}\right) \hat{h}_{k+1}^{*}\left(j+2^{k}\right)=0,
\end{aligned}
$$

for $j=0,1, \ldots, 2^{k}-1$.

Note that the projection operator $P_{k}^{*}: X_{2 \pi}^{*} \rightarrow V_{k}^{*}$ corresponding to the bounded linear functionals $v_{k, j} \in X_{2 \pi}^{* *}, j=0,1, \ldots, 2^{k}-1$, defined by

$$
P_{k}^{*} g^{*}:=\sum_{j=0}^{2^{k}-1}\left\langle v_{k, j}, g^{*}\right\rangle v_{k, j}^{*}, \quad g^{*} \in X_{2 \pi}^{*},
$$

is the adjoint of $P_{k}$. Indeed, if $f \in X_{2 \pi}$ and $g^{*} \in X_{2 \pi}^{*}$, then

$$
\left\langle P_{k} f, g^{*}\right\rangle=\sum_{j=0}^{2^{k}-1}\left\langle f, v_{k, j}^{*}\right\rangle\left\langle v_{k, j}, g^{*}\right\rangle=\left\langle f, P_{k}^{*} g^{*}\right\rangle
$$

Proposition 6.2. Suppose that $\left\{V_{k}\right\}_{k \geq 0}$ is a multiresolution of $X_{2 \pi}$, and $P_{k}: X_{2 \pi} \rightarrow V_{k}$, $k=0,1, \ldots$, is a sequence of projection operators satisfying (4.19), (4.28), (4.29) and (5.9). Then $\left\{V_{k}^{*}\right\}_{k \geq 0}$ is a multiresolution of

$$
Y_{2 \pi}^{*}:=\overline{\bigcup_{k \geq 0} V_{k}^{*}}
$$

Further, if $X_{2 \pi}=L_{2 \pi}^{p}, 1<p<\infty$, then

$$
Y_{2 \pi}^{*}=L_{2 \pi}^{q}
$$

where $1 / p+1 / q=1$.

Proof. Let

$$
\phi_{k}^{*}:=\frac{1}{\sqrt{2^{k}}} \sum_{j=0}^{2^{k}-1} v_{k, j}^{*}
$$

By (4.20),

$$
T_{k}^{\ell} \phi_{k}^{*}=\frac{1}{\sqrt{2^{k}}} \sum_{j=0}^{z^{k}-1} \omega_{k}^{\ell j} v_{k, j}^{*}
$$


By (4.13), $\left\{v_{k, j}^{*}: j=0,1, \ldots, 2^{k}-1\right\}$ is linearly independent. Hence $\left\{T_{k}^{\ell} \phi_{k}^{*}: \ell=\right.$ $\left.0,1, \ldots, 2^{k}-1\right\}$ is a basis for $V_{k}^{*}$. Therefore MR1 is satisfied. The condition MR2 follows from (6.9), and MR3 for $Y_{2 \pi}^{*}$ follows from the definition (6.14).

To prove (6.15), take any $f \in L_{2 \pi}^{p}$. Then (4.28), (4.29) and the MR3 condition for $\left\{V_{k}\right\}_{k \geq 0}$ imply that $\left\|\left(I-P_{k}\right) f\right\| \rightarrow 0$ as $k \rightarrow \infty$. If $g^{*} \in L_{2 \pi}^{q}$,

$$
\left|\left\langle f,\left(I^{*}-P_{k}^{*}\right) g^{*}\right\rangle\right|=\left|\left\langle\left(I-P_{k}\right) f, g^{*}\right\rangle\right| \leq\left\|\left(I-P_{k}\right) f\right\|\left\|g^{*}\right\| \rightarrow 0 \text { as } k \rightarrow \infty .
$$

Thus $P_{k}^{*} g^{*}$ converges to $g^{*}$ weakly. Hence $U_{k \geq 0} V_{k}^{*}$ is weakly dense in $L_{2 \pi}^{q}$. Since $U_{k \geq 0} V_{k}^{*}$ is convex, it follows that it is also strongly dense in $L_{2 \pi}^{q}$.

If $\left\{P_{k}\right\}_{k \geq 0}$ satisfies (4.19), (4.28) and (4.29), then

$$
P_{k}^{*} T_{k}=T_{k} P_{k}^{*}
$$

$\left\{P_{k}^{*}\right\}_{k \geq 0}$ is uniformly bounded and

$$
P_{k}^{*} P_{k+1}^{*}=P_{k+1}^{*} P_{k}^{*}=P_{k}^{*},
$$

i.e. $\left\{P_{k}^{*}\right\}_{k \geq 0}$ also satisfies (4.19), (4.28) and (4.29). Let

$$
W_{k}^{*}:=V_{k+1}^{*} \cap \operatorname{Ker}\left(P_{k}^{*}\right)
$$

Then

$$
Y_{2 \pi}^{*}=V_{0}^{*} \oplus W_{0}^{*} \oplus W_{1}^{*} \oplus \cdots
$$

is a Schauder decomposition of $Y_{2 \pi}^{*}$. Further, for $j=0,1, \ldots, 2^{k}-1,(4.7)$ gives

$$
\left\langle v_{k, j}, v_{k+1, \ell}^{*}\right\rangle= \begin{cases}0, & \ell \neq j, j+2^{k}, \\ \frac{1}{\sqrt{2}} \hat{h}_{k+1}(\ell), & \ell=j, j+2^{k}\end{cases}
$$

which shows that the functionals $v_{k, j} \in Y_{2 \pi}^{* *}$ corresponding to the projection $P_{k}^{*}$ satisfy the condition (5.8) of Theorem 5.1.

The construction in the proof of Theorem 5.1 gives sequences $\hat{g}_{k+1}^{*} \in S\left(2^{k+1}\right)$ such that for $j=0,1, \ldots, 2^{k}-1$,

(6c) if $\hat{h}_{k+1}(j) \neq 0$, then $\hat{g}_{k+1}^{*}\left(j+2^{k}\right)$ is an arbitrary nonzero number and

$$
\hat{g}_{k+1}^{*}(j):=\frac{-\hat{g}_{k+1}^{*}\left(j+2^{k}\right) \hat{h}_{k+1}\left(j+2^{k}\right)}{\hat{h}_{k+1}(j)}
$$

(6d) if $\hat{h}_{k+1}(j)=0$, then $\hat{g}_{k+1}^{*}(j)$ is an arbitrary nonzero number and $\hat{g}_{k+1}^{*}\left(j+2^{k}\right):=0$. 
The relation (6.11) is self-dual, while the dual of (6.12) is

$$
\hat{g}_{k+1}^{*}(j) \hat{h}_{k+1}(j)+\hat{g}_{k+1}^{*}\left(j+2^{k}\right) \hat{h}_{k+1}\left(j+2^{k}\right)=0
$$

for $j=0,1, \ldots, 2^{k}-1$, which follows from the above construction.

For $k=0,1, \ldots$, let $\psi_{k}^{*} \in Y_{2 \pi}^{*}$ be defined via its Fourier coefficients:

$$
\hat{\psi}_{k}^{*}\left(-j+2^{k+1} p\right):=\hat{g}_{k+1}^{*}(j) \hat{\phi}_{k+1}^{*}\left(-j+2^{k+1} p\right)
$$

for all $p \in \mathbf{Z}, j=0,1, \ldots, 2^{k+1}-1$. Define

$$
u_{k, j}^{*}:=\frac{1}{\sqrt{2^{k}}} \sum_{\ell=0}^{2^{k}-1} \omega_{k}^{-j \ell} T_{k}^{\ell} \psi_{k}^{*}, \quad j=0,1, \ldots, 2^{k}-1,
$$

equivalently,

$$
T_{k}^{\ell} \psi_{k}^{*}=\frac{1}{\sqrt{2^{k}}} \sum_{j=0}^{2^{k}-1} \omega_{k}^{\ell j} u_{k, j}^{*}, \quad \ell=0,1, \ldots, 2^{k}-1
$$

The functions $\psi_{k}$ and $\psi_{k}^{*}$ which generate wavelet bases for $W_{k}$ and $W_{k}^{*}$ are obtained from the sequences $\hat{g}_{k+1}$ and $\hat{g}_{k+1}^{*}$ which satisfy the general conditions (6a), (6b) and (6c), (6d) respectively. We shall show that a judicious choice of $\hat{g}_{k+1}$ and $\hat{g}_{k+1}^{*}$ produces functions $\psi_{k}$ and $\psi_{k}^{*}$ which generate a biorthogonal system.

Lemma 6.1. For $j=0,1, \ldots, 2^{k}-1$, let

$$
\hat{g}_{k+1}(j):=-\hat{h}_{k+1}^{*}\left(j+2^{k}\right), \quad \hat{g}_{k+1}\left(j+2^{k}\right):=\hat{h}_{k+1}^{*}(j)
$$

and

$$
\hat{g}_{k+1}^{*}(j):=-\hat{h}_{k+1}\left(j+2^{k}\right), \quad \hat{g}_{k+1}^{*}\left(j+2^{k}\right):=\hat{h}_{k+1}(j)
$$

Then $\hat{g}_{k+1}$ satisfies (6a) and (6b), and $\hat{g}_{k+1}^{*}$ satisfies (6c) and (6d). Further, for $j=0,1, \ldots, 2^{k}-1$,

$$
\hat{g}_{k+1}(j) \hat{g}_{k+1}^{*}(j)+\hat{g}_{k+1}\left(j+2^{k}\right) \hat{g}_{k+1}^{*}\left(j+2^{k}\right)=2
$$

Proof. It is straightforward to verify that $\hat{g}_{k+1}$ and $\hat{g}_{k+1}^{*}$ defined by (6.22) and (6.23) satisfy (6a), (6b) and (6c), (6d) respectively. The relation (6.24) follows from (6.22), (6.23) and (6.11). 
Theorem 6.1. Suppose that $\left\{V_{k}\right\}_{k \geq 0}$ is a multiresolution of $X_{2 \pi}, P_{k}: X_{2 \pi} \rightarrow V_{k}$, $k=0,1, \ldots$, is a sequence of projection operators satisfying (4.19), (4.28), (4.29) and (5.9), and $\hat{g}_{k+1}$ and $\hat{g}_{k+1}^{*}$ satisfy (6.22) and (6.23). Then

$$
\left\{\left\{v_{0,0}, u_{k, j}\right\},\left\{v_{0,0}^{*}, u_{k, j}^{*}\right\}: k=0,1, \ldots, j=0,1, \ldots, 2^{k}-1\right\}
$$

is a biorthogonal system.

Proof. The relation (5.5) and its dual,

$$
u_{k, j}^{*}=\frac{1}{\sqrt{2}} \hat{g}_{k+1}^{*}(j) v_{k+1, j}^{*}+\frac{1}{\sqrt{2}} \hat{g}_{k+1}^{*}\left(j+2^{k}\right) v_{k+1, j+2^{k}}^{*}
$$

give

$$
\left\langle u_{k, j}, u_{k, \ell}^{*}\right\rangle=0 \text { for } j \neq \ell
$$

and

$$
\left\langle u_{k, j}, u_{k, j}^{*}\right\rangle=\frac{1}{2}\left(\hat{g}_{k+1}(j) \hat{g}_{k+1}^{*}(j)+\hat{g}_{k+1}\left(j+2^{k}\right) \hat{g}_{k+1}^{*}\left(j+2^{k}\right)\right)=1,
$$

by (6.24). Hence

$$
\left\langle u_{k, j}, u_{k, \ell}^{*}\right\rangle=\delta_{j, \ell}, \quad j, \ell=0,1, \ldots, 2^{k}-1 .
$$

Similarly, (5.5), (6.10) and (6.12) lead to

$$
\left\langle u_{k, j}, v_{k, \ell}^{*}\right\rangle=0, \quad j, \ell=0,1, \ldots, 2^{k}-1,
$$

while (4.7), (6.25) and (6.18) give

$$
\left\langle v_{k, j}, u_{k, \ell}^{*}\right\rangle=0, \quad j, \ell=0,1, \ldots, 2^{k}-1 .
$$

Now if $k<m$, then $V_{k+1} \subset V_{m}$. Hence $W_{k} \subset V_{m}$, and (6.28) implies that

$$
\left\langle u_{k, j}, u_{m, \ell}^{*}\right\rangle=0, \quad j=0,1, \ldots, 2^{k}-1, \ell=0,1, \ldots, 2^{m}-1 .
$$

Similarly, if $k>m$, (6.27) implies (6.29). The result follows from (6.26) and (6.29).

Corollary 6.1. If the conditions of Theorem 6.1 are satisfied, then

$$
\left\{\left\{\phi_{0}, T_{k}^{\ell} \psi_{k}\right\},\left\{\phi_{0}^{*}, T_{k}^{\ell} \psi_{k}^{*}\right\}: k=0,1, \ldots, \ell=0,1, \ldots, 2^{k}-1\right\}
$$

is a biorthogonal system. 
Proof. The result follows from Theorem 6.1, (5.3) and (6.21).

\section{Projections and decompositions and reconstruction algorithms}

Suppose that $\left\{V_{k}\right\}_{k \geq 0}$ is a multiresolution of $X_{2 \pi}, P_{k}: X_{2 \pi} \rightarrow V_{k}, k=0,1, \ldots$, a sequence of projections on $V_{k}$ satisfying (4.19), (4.28), (4.29) and (5.9), and $Q_{k}:=P_{k+1}-P_{k}$. Then $Q_{k}$ is a projection on $W_{k}, k=0,1, \ldots$, and

$$
V_{k+1}=V_{k} \oplus W_{k}, \quad k=0,1, \ldots,
$$

where

$$
W_{k}=\operatorname{Ker}\left(\left.P_{k}\right|_{\nu_{k+1}}\right)=V_{k+1} \bigcap \operatorname{Ker}\left(P_{k}\right)=Q_{k}\left(X_{2 \pi}\right)
$$

The space $W_{k}$ has a basis generated by $T_{k}^{\ell} \psi_{k}, \ell=0,1, \ldots, 2^{k}-1$. Also,

$$
W_{k}=\operatorname{span}\left\{u_{k, j}: j=0,1, \ldots, 2^{k}-1\right\},
$$

where $\left\{u_{k, j}: j=0,1, \ldots, 2^{k}-1\right\}$ is the rotation of $\left\{T_{k}^{\ell} \psi_{k}: \ell=0,1, \ldots, 2^{k}-1\right\}$ by the Fourier matrix with a suitable normalization. Therefore $Q_{k}$ can be expressed as

$$
Q_{k} f=\sum_{j=0}^{2^{k}-1}\left(f, u_{k, j}^{*}\right) u_{k, j}, \quad f \in X_{2 \pi},
$$

where $\left\{u_{k, j}^{*}: j=0,1, \ldots, 2^{k}-1\right\}$ is the biorthogonal basis for $W_{k}^{*}$.

For $j=0,1, \ldots, 2^{k+1}-1, \ell=0,1, \ldots, 2^{k}-1$, define

$$
\mu_{j, \ell}^{k}=\left(v_{k+1, j}, u_{k, \ell}^{*}\right)
$$

Then (4.7) and (5.5) lead to

$$
\begin{aligned}
& \hat{h}_{k+1}(j) \mu_{j, \ell}^{k}+\hat{h}_{k+1}\left(j+2^{k}\right) \mu_{j+2^{k}, \ell}^{k}=0, \\
& \hat{g}_{k+1}(j) \mu_{j, \ell}^{k}+\hat{g}_{k+1}\left(j+2^{k}\right) \mu_{j+2^{k}, \ell}^{k}=\sqrt{2} \delta_{j, \ell}, \quad j, \ell=0,1, \ldots, 2^{k}-1 .
\end{aligned}
$$

Note that (7.3) and (7.4) are analogous to (5.16) and (5.15) where the roles of $\hat{h}_{k+1}$ and $\hat{g}_{k+1}$ are interchanged, and $\beta_{j, \ell}^{k}:=\left\langle v_{k+1, j}, v_{k, \ell}^{*}\right\rangle$ is replaced by $\mu_{j, \ell}^{k}$.

Since $v_{k, J} \neq 0$,

$$
\left|\hat{h}_{k+1}(j)\right|+\left|\hat{h}_{k+1}\left(j+2^{k}\right)\right| \neq 0
$$

This implies, as before, that 


$$
\mu_{j, \ell}^{k}=\mu_{j+2^{k}, \ell}^{k}=0, \quad j, \ell=0,1, \ldots, 2^{k}-1 \text { and } j \neq \ell .
$$

Thus (7.3) and (7.4) become

$$
\begin{aligned}
& \hat{h}_{k+1}(j) \mu_{j, j}^{k}+\hat{h}_{k+1}\left(j+2^{k}\right) \mu_{j+2^{k}, j}^{k}=0, \\
& \hat{g}_{k+1}(j) \mu_{j, j}^{k}+\hat{g}_{k+1}\left(j+2^{k}\right) \mu_{j+2^{k}, j}^{k}=\sqrt{2}, \quad j=0,1, \ldots, 2^{k}-1 .
\end{aligned}
$$

Lemma 7.1. The linear system (7.6) and (7.7) is nonsingular for each $j=0,1, \ldots$, $2^{k}-1$.

Proof. Since

$$
\left|\hat{h}_{k+1}(j)\right|+\left|\hat{h}_{k+1}\left(j+2^{k}\right)\right| \neq 0
$$

and

$$
\left|\hat{g}_{k+1}(j)\right|+\left|\hat{g}_{k+1}\left(j+2^{k}\right)\right| \neq 0,
$$

each of the rows of the matrix of coefficients is nonzero. If the linear system is singular, then there exists a nonzero constant $c$ such that

$$
\left(\hat{h}_{k+1}(j), \hat{h}_{k+1}\left(j+2^{k}\right)\right)=c\left(\hat{g}_{k+1}(j), \hat{g}_{k+1}\left(j+2^{k}\right)\right) .
$$

Thus (7.6) becomes

$$
c\left(\hat{g}_{k+1}(j) \mu_{j, j}^{k}+\hat{g}_{k+1}\left(j+2^{k}\right) \mu_{j+2^{k}, j}^{k}\right)=0,
$$

which implies that $c=0$, a contradiction.

Solving the linear system (7.6) and (7.7) gives

$$
\begin{aligned}
\mu_{j, j}^{k} & =\frac{-\sqrt{2} \hat{h}_{k+1}\left(j+2^{k}\right)}{\hat{h}_{k+1}(j) \hat{g}_{k+1}\left(j+2^{k}\right)-\hat{h}_{k+1}\left(j+2^{k}\right) \hat{g}_{k+1}(j)}, \\
\mu_{j+2^{k} . J}^{k} & =\frac{\sqrt{2} \hat{h}_{k+1}(j)}{\hat{h}_{k+1}(j) \hat{g}_{k+1}\left(j+2^{k}\right)-\hat{h}_{k+1}\left(j+2^{k}\right) \hat{g}_{k+1}(j)} .
\end{aligned}
$$

If $\hat{g}_{k+1}$ and $\hat{g}_{k+1}^{*}$ satisfy (6.22) and (6.23), then using (6.11), the expressions in (7.8) and (7.9) simplify to give 


$$
\hat{g}_{k+1}^{*}(j)=\left\{\begin{array}{l}
\sqrt{2} \mu_{j, j}^{k}, j=0,1, \ldots, 2^{k}-1, \\
\sqrt{2} \mu_{j, j-2^{k}}^{k}, j=2^{k}, \ldots, 2^{k+1}-1
\end{array}\right.
$$

Suppose that for $k=0,1, \ldots, f_{k+1} \in V_{k+1}$, and that

$$
f_{k+1}=f_{k}+d_{k}, \quad f_{k} \in V_{k}, d_{k} \in W_{k},
$$

where

$$
\begin{aligned}
& f_{k}=\sum_{j=0}^{2^{k}-1} \hat{s}_{k}(j) v_{k, j}, \\
& d_{k}=\sum_{j=0}^{2^{k}-1} \hat{t}_{k}(j) u_{k, j},
\end{aligned}
$$

and $\hat{s}_{k}, \hat{t}_{k} \in S\left(2^{k}\right)$. Then a familiar argument leads to the following frequency-based reconstruction and decomposition algorithms (see [9]):

$$
\begin{gathered}
\hat{s}_{k+1}(j)=\frac{1}{\sqrt{2}} \hat{h}_{k+1}(j) \hat{s}_{k}(j)+\frac{1}{\sqrt{2}} \hat{g}_{k+1}(j) \hat{t}_{k}(j), \quad j=0,1, \ldots, 2^{k+1}-1, \\
\hat{s}_{k}(j)=\frac{1}{\sqrt{2}} \hat{h}_{k+1}^{*}(j) \hat{s}_{k+1}(j)+\frac{1}{\sqrt{2}} \hat{h}_{k+1}^{*}\left(j+2^{k}\right) \hat{s}_{k+1}\left(j+2^{k}\right) \\
\hat{t}_{k}(j)=\frac{1}{\sqrt{2}} \hat{g}_{k+1}^{*}(j) \hat{s}_{k+1}(j)+\frac{1}{\sqrt{2}} \hat{g}_{k+1}^{*}\left(j+2^{k}\right) \hat{s}_{k+1}\left(j+2^{k}\right)
\end{gathered}
$$

for $j=0,1, \ldots, 2^{k}-1$.

\section{Examples}

8.1. Orthogonal projections and multiresolutions in $L_{2 \pi}^{2}$. Let $\left\{V_{k}\right\}_{k \geq 0}$ be a multiresolution of $L_{2 \pi}^{2}$ generated by $\phi_{k}, k \geq 0$, constructed as in Corollary 3.1. Let $P_{k}: L_{2 \pi}^{2} \rightarrow L_{2 \pi}^{2}$ be the orthogonal projection on $V_{k}$ for $k \geq 0$. Then

$$
P_{k} f:=\sum_{j=0}^{2^{k}-1} \frac{\left(f, v_{k, j}\right) v_{k, j}}{\left\|v_{k, j}\right\|^{2}}
$$

which satisfies (4.19), (4.28), (4.29) and (5.9) with $\left\|P_{k}\right\| \leq 1$. A straightforward computation gives 


$$
\begin{aligned}
\beta_{j, j}^{k} & =\frac{\overline{\hat{h}_{k+1}(j)}\left\|v_{k+1, j}\right\|^{2}}{\sqrt{2}\left\|v_{k, j}\right\|^{2}}, \\
\beta_{j+2^{k}, j}^{k} & =\frac{\overline{\hat{h}_{k+1}\left(j+2^{k}\right)}\left\|v_{k+1, j+2^{k}}\right\|^{2}}{\sqrt{2}\left\|v_{k, j}\right\|^{2}},
\end{aligned}
$$

for $j=0,1, \ldots, 2^{k}-1$. By our construction,

(a1) if $\beta_{j, j}^{k} \neq 0$, equivalently $\hat{h}_{k+1}(j) \neq 0$, we let $\hat{g}_{k+1}\left(j+2^{k}\right)$ be an arbitrary nonzero number and set

$$
\hat{g}_{k+1}(j):=\frac{-\hat{g}_{k+1}\left(j+2^{k}\right) \overline{\hat{h}_{k+1}\left(j+2^{k}\right)}\left\|v_{k+1, j+2^{k}}\right\|^{2}}{\overline{\hat{h}_{k+1}(j)}\left\|v_{k+1, j}\right\|^{2}} ;
$$

(b1) if $\beta_{j, j}^{k}=0$, equivalently $\hat{h}_{k+1}(j)=0$, we let $\hat{g}_{k+1}(j)$ be an arbitrary nonzero number and set $\hat{g}_{k+1}\left(j+2^{k}\right):=0$.

The corresponding wavelets $\psi_{k}$ are obtained from $\hat{g}_{k+1}$ using (5.1). Note that the sequences $\hat{g}_{k+1}$ and hence the corresponding wavelets constructed in [9] are special cases of (a1) and (b1) above.

8.2 Interpolatory projections and multiresolutions in $C_{2 \pi}$. Let $\phi$ be a refinable, continuous and compactly supported function on $\mathbf{R}$ satisfying the following conditions:

$$
\begin{gathered}
\sum_{n \in \mathbf{Z}} \phi(n) e^{i n x} \neq 0 \text { for all } x \in \mathbf{R}, \\
\int_{-\infty}^{\infty} \phi(x) d x \neq 0
\end{gathered}
$$

and

$$
\hat{\phi}(u)=O\left(|u|^{-1-\epsilon}\right) \text { as }|u| \rightarrow \infty,
$$

for some $\epsilon>0$. Define a function $L$ by

$$
L(x):=\sum_{v \in \mathbf{Z}} \omega_{v} \phi(x-v)
$$

where $\omega_{v}, v \in \mathbf{Z}$, are the coefficients of the Laurent series 


$$
\sum_{v \in Z} \omega_{v} z^{v}:=\frac{1}{\sum_{n \in Z} \phi(n) z^{n}}, \quad|z|=1
$$

For $k \geq 0$, periodize the functions $\phi$ and $L$ by

$$
\phi_{k}(x):=\sum_{n \in \mathbf{Z}} \phi\left(\frac{2^{k}}{2 \pi}(x+2 \pi n)\right)
$$

and

$$
L_{k}(x):=\sum_{n \in \mathbb{Z}} L\left(\frac{2^{k}}{2 \pi}(x+2 \pi n)\right)
$$

Then it follows from Proposition 2.1, Corollary 2.1 and the Poisson's summation formula that $\phi_{k}, k \geq 0$, generate a multiresolution $\left\{V_{k}\right\}_{k \geq 0}$ of $C_{2 \pi}$ with $v_{k, j}(0) \neq 0$ for all $j=0,1, \ldots, 2^{k}-1$, and $k \geq 0$. Let $P_{k}: C_{2 \pi} \rightarrow C_{2 \pi}$ be the interpolatory projection on $V_{k}$ for $k \geq 0$. Then $P_{k}$ is given by (4.15), where

$$
\left\langle f, v_{k, j}^{*}\right\rangle:=\frac{1}{2^{k} v_{k, j}(0)} \sum_{l=0}^{2^{k}-1} f\left(\frac{2 \pi l}{2^{k}}\right) \omega_{k}^{-j l}, \quad j=0,1, \ldots, 2^{k}-1
$$

The projection $P_{k}$ can be expressed by

$$
P_{k} f=\sum_{j=0}^{2^{k}-1} f\left(\frac{2 \pi j}{2^{k}}\right) L_{k}\left(-\frac{2 \pi j}{2^{k}}\right)
$$

which satisfies (4.28) with

$$
\left\|P_{k}\right\| \leq \max _{x \in[0,1]} \sum_{n \in \mathbf{Z}}|L(x-n)|
$$

Further, (4.15) and (8.10) yield (4.19), (4.29) and (5.9) with

$$
\begin{aligned}
\beta_{j, J}^{k} & =\frac{v_{k+1, j}(0)}{v_{k, j}(0)}, \\
\beta_{j+2^{k}, j}^{k} & =\frac{v_{k+1, j+2^{k}}(0)}{v_{k, j}(0)},
\end{aligned}
$$

for $j=0,1, \ldots, 2^{k}-1$. The values $\beta_{j, j}^{k}$ and $\beta_{j+2^{k}, j}^{k}$ are both nonzero. By our construction, we let $\hat{g}_{k+1}\left(j+2^{k}\right)$ be an arbitrary nonzero number and set 


$$
\hat{g}_{k+1}(j):=\frac{-\hat{g}_{k+1}\left(j+2^{k}\right) v_{k+1, j+2^{k}}(0)}{v_{k+1, j}(0)}
$$

Then the corresponding wavelets $\psi_{k}$ are obtained from $\hat{g}_{k+1}$ using (5.1). This is the periodic analogue of the interpolating wavelets studied in [2], [7] and [12].

Remark 3. After the completion of our paper, it was brought to our attention the existence of [1], [4] and [5] where the abstract theory of multiscale projections, which overlaps with part of our paper, was treated in more generality. Our approach makes use of orthogonal bases of "orthogonal splines" which simplifies the analysis in the periodic case.

Acknowledgement. The authors would like to thank the anonymous referee for his helpful suggestions, which improve the results of this paper.

\section{REFERENCES}

1. J. M. Carnicer, W. Dahmen and J. M. Peña, Local decomposition of refinable spaces and wavelets, Appl. Comput. Harmonic Anal. 3 (1996), 127-153.

2. C. K. CHUI and C. LI, Dyadic affine decompositions and functional wavelet transformations, SIAM J. Math. Anal. 27 (1996), 865-890.

3. C. K. ChUi and N. H. Mhaskar, On trigonometric wavelets, Constr. Approx. 9 (1993), $167-190$.

4. W. DAHMEN, Some remarks on multiscale transformations, stability and biorthogonality, in Curves and Surfaces II (P. J. Laurent, A. Le Méhauté and L. L. Schumaker, eds., Academic Press, San Diego, 1994), 157-188.

5. W. Dahmen, Stability of multiscale transformations, preprint.

6. I. DaUbechIEs, Orthonormal bases of compactly supported wavelets, Comm. Pure Appl. Math. 41 (1988), 909-996.

7. D. L. Donoнo, Interpolating wavelet transform, preprint.

8. Y. KatZnelson, An Introduction to Harmonic Analysis (Dover, New York, 1976).

9. Y. W. KоH, S. L. LeE and H. H. TAN, Periodic orthogonal splines and wavelets, Appl. Comput. Harmonic Anal. 2 (1995), 201-218.

10. S. Mallat, Multiresolution approximations and wavelet orthonormal bases of $L^{2}(\mathbf{R})$, Trans. Amer. Math. Soc. 315 (1989), 69-87.

11. J. T. MARTI, Introduction to the Theory of Bases (Springer-Verlag, New York, 1969).

12. C. A. Micchelli, Using refinement equation for the construction of pre-wavelets, Numerical Algorithm 1 (1991), 75-116.

13. F. J. Narcowich and J. D. WARD, Wavelets associated with periodic basis functions, Appl. Comput. Harmonic Anal. 3 (1996), 40-56. 
14. G. Plonka and M. TASCHE, A unified approach to periodic wavelets, in Wavelets: Theory. Algorithms, and Applications (C. K. Chui, L. Montefusco and L. Puccio, eds., Academic Press, New York, 1994), 137-151.

15. H. RerTeR, Classical Harmonic Analysis and Locally Compact Groups (Oxford University Press, London, 1968).

Department OF Mathematics

National UNIVERSiTY OF SINGAPORE

10 Kent Ridge Crescent

SINGAPORE 119260

REPUBLIC OF SINGAPORE 